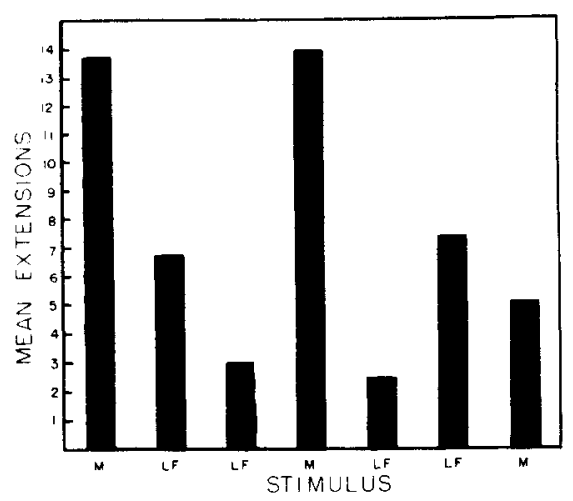

Fig. 2. Mean number of gill cover extensions during $15 \mathrm{~min}$ by 10 Ss. Each observation was separated by 1 week. $M=$ Mirror observation $; \mathbf{L F}=$ live male fish present in adjacent tank during observation.

but overall GCEs were almost equal in Conditions 1 and 2. Superficially, this would seem to indicate that the aggressive display of male Bettas is little affected by a female's presence. However, while not affecting total number of GCEs, the female clearly altered the distribution of displays in each area of the tank. In the female's absence, Ss displayed primarily before the mirror; with a female, displays were about equally distributed among the three areas of the tank. When the female was absent the center area was an "escape" region, devoid of stimuli, and there were almost no displays there. Ss displayed to the female when she was present in the center area, and performed fewer GCEs to the mirror image; display frequency to the male stimulus fish was maintained. Displaying males attempted to break into the breeder net, and although these sexual displays were similar in kind to aggressive displays made to males (Kühme, 1963), movements were slower, with less thrashing and more biting.

\section{EXPERIMENT 2}

The preference for mirror image stimulation found in Experiment 1, and the greater frequency of gill cover extensions to mirror images, could conceivably be limited to situations in which $\mathrm{S}$ is free to move between a mirror, a conspecific male, and no stimulation. Accordingly, the frequency of GCE to mirrors and conspecific males was investigated by presenting these stimuli to male Bettas for 15 -min periods separated by intervals of 1 week, in a situation where Ss were not free to move away from them.

\section{Method}

Ten naive male Bettas were isolated for 2 weeks prior to experimentation. Observa. tions were made while Ss were in their home containers (500-ml rectangular glass jars). A mirror or a stimulus fish in a similar jar was placed against one wall of S's container of $15 \mathrm{~min}$ and the frequency of GCEs was recorded. Observations were made at weekly intervals between 2 and 4:30 p.m. in the following order: mirror, habituated male, habituated male, mirror, unhabituated male, unhabituated male, mirror.

\section{Results}

Gill cover extensions were performed more frequently to mirrors than to live males during the first four observations $(\mathrm{t}=2.4, \mathrm{p}<.01)$. A significant positive correlation was found between the GCE frequency to live fish in Sessions 2 and 3 $(\mathrm{r}=0.62, \mathrm{p}<.05)$, but not between GCEs to mirror images in Sessions 1 and 4 $(r=0.33, p>.05)$. The sum of GCEs in the two mirror sessions was positively related to the sum of GCEs in the sessions with live males as stimuli $(r=0.70, p<.05)$.

In the last four sessions no difference was found between displays to mirrors and live fish, presumably because of diminished responding to the mirror in the final observation. Again, a positive correlation between GCEs to live fish in Observations 5 and 6 was found $(r=0.84, p<.01)$ but not between Mirror Sessions 4 and $7(r=0.48$, $p>.05)$. Waning of responsiveness to mirrors was found in this experiment, even though observations were always separated by 1 week: Only two Ss displayed to mirrors in Observation 7, while nine did so in Observations 1 and 4. Evidence that some Ss showed waning responsiveness to live fish was also found: In Observations 2, 3,5, and 6 there were $7,6,7$, and 3 Ss which showed displays, respectively.

\section{Discussion}

Quite clearly, the increased effectiveness of mirrors over live males as display-eliciting stimuli is not limited to preference situations. The responsiveness of a given $S$ was more consistent to live fish than toward mirror image opponents, due perhaps to the fact that a mirror image opponent behaves consistently only to the extent that S's behavior is consistent while a live opponent may behave consistently independently of S. Gallup (1968) has suggested that the effectiveness of mirror image stimulation may be due to its novelty. If this were the case, one might expect a more rapid habituation to mirror images than to live fish, the responsiveness to the latter being due presumably to something besides novelty. No clear evidence of such a differential waning has been found in these experiments or in previous work with $B$. splendens (Baenninger, 1966, 1968).

\section{REFERENCES}

BAENNINGER, R. Waning of aggressive motivation in Betta splendens. Psychonomic Science, 1966, 4, 241-242.

BAENNINGER, R. Catechol amines and social relations in Siamese fighting fish. Animal Behaviour, 1968, 16, 442-447.

CLAYTON, F. L., \& HINDE, R. A. The habituation and recovery of aggressive display in Betta splendens. Behaviour, 1968, 30,1.

GALLUP, G. G. Mirror image stimulation. Psychological Bulletin, 1968, 70, 782-793.

KÜHME, W. Verhaltensstudien am maulbrutenden (Betta anabantoides Bleeker) und am nestbauenden Kampffisch (Betta splendens Regan). Zeitschrift für Tierpsychologie, 1963, 18, 33-55.

NOTE

1. A grant-in-aid from Temple University provided funds for the conduct of this research. Our thanks to Garrett Bergman for assistance in the observation of Ss in Experiment 1.

\title{
Reversal learning in the sow bug'
}

\section{J. W. McDANIEL, Tulane University, New Orleans, La. 70118}

Three groups of four sow bugs each were taught a tuming response in a simple $T$-maze. One group, acting as a control, learned a single turning response. The other two groups were taught successive reversals according to a learning criterion or a trials criterion. Under the conditions of this study, sow bugs demonstrated statistically significant improvement in successive reversal learning.

Previous research has shown that sow bugs are capable of learning only relatively simple tasks. Frost (1967) has reported that sow bugs can readily master a single turning response in a simple $\mathrm{T}$-maze. Thompson (1957), on the other hand, found sow bugs unable to show progressive improvement in habit reversal over a number of days. Thompson ran each $S$ eight trials per day in a simple T-maze using electric shock as a negative reinforcer and a dark, damp goal box as the positive reinforcer. After reaching a criterion of seven out of eight responses correct, the correct arm of the maze was reversed until criterion was again reached. Seven Ss were trained until each had completed eight reversals; no significant improvement over reversals was shown. Ratner \& Denny $(1964$, p. 634) suggested that under more favorable circumstances sow bugs could probably demonstrate reversal learning. Since most Isopods are negatively thermotaxic, it was decided for the present study to use a more salient negative reinforcer, extreme heat, and also to mass practice during training. 

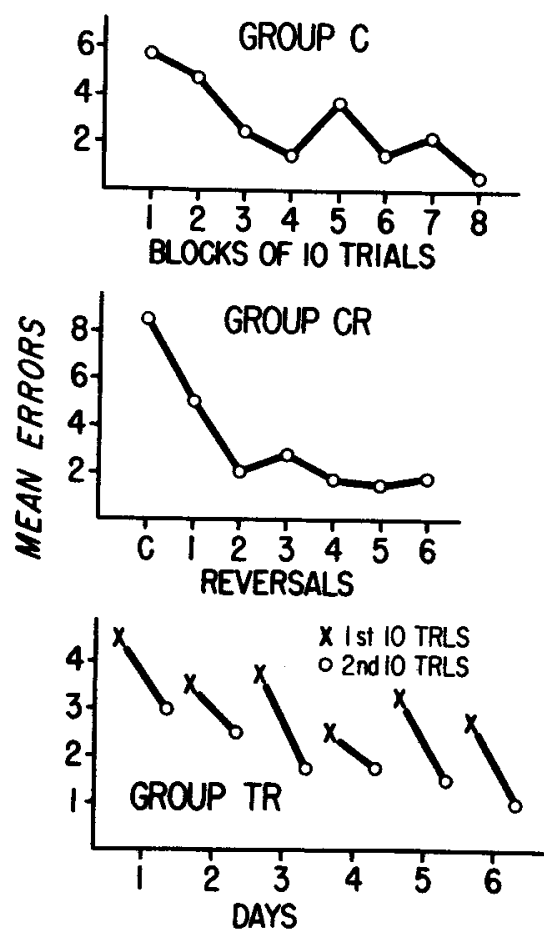

Fig. 1. Mean errors for three groups of sow bugs on a maze-learning task. Group $C$ learned a single turning response; Group $\mathbf{C R}$ was reversed after reaching a criterion of five successive correct responses; Group TR was reversed after every 10 trials.

\section{METHOD}

Twelve common sow bugs (Isopod Crustacea of the genus Porcellio) were unsystematically divided into three groups of four Ss each: Group C, simple learning control; Group CR, criterion reversal; and Group TR, trials reversal. The apparatus consisted of a simple black plastic T-maze with an $8-\mathrm{cm}$ stem and two $4-\mathrm{cm}$ arms. The alleys were $1 \mathrm{~cm}$ wide and $2 \mathrm{~cm}$ deep. Negative reinforcement was provided by a 23-W, 115.V pencil soldering iron with a rated tip temperature of $750 \mathrm{deg} F$. Preliminary research had shown that the minimum distance a sow bug would approach to this iron was $5 \mathrm{~cm}$. Positive reinforcement was escape from the maze into a dark, damp match box.

The basic procedure was the same for all groups. The $\mathrm{S}$ was introduced into the stem of the maze which was then closed to prevent escape. At the choice point a response was defined as the complete entry of an $S$ into an arm. If the response was correct the $S$ was allowed to continue and enter the goal box. If incorrect, the soldering iron was inserted into the maze approximately $3 \mathrm{~cm}$ ahead of the $S$ and held there until the $S$ turned around and moved away. An $\mathrm{S}$ was not removed from the maze until he had made the correct response.

Groups $C$ and CR were given 20 trials per day for 4 days, a total of 80 trials. Group TR was given 20 trials per day for 6 days, 120 trials. The intertrial interval for all groups was approximately $3 \mathrm{~min}$. The first trial of the first day for each $S$ was defined as incorrect, and the negative reinforcer was applied. Thus, each $\mathrm{S}$ determined which arm of the maze was initially correct. This was done to control for turning preference without introducing a number of initial unreinforced trials into training. The Ss were run in squads of four, and the three other Ss were run between any two trials for a given S.

Group C was required to learn a single turning response throughout all trials. Group CR was required to learn successive reversals of the turning response; the correct arm of the maze was reversed after an $S$ had reached a criterion of five successive correct responses. For Group TR the correct arm of the maze was reversed after each 10 trials. These Ss were given two reversals per day for 6 days.

\section{RESULTS}

Both Groups $\mathrm{C}$ and $\mathrm{CR}$ showed a significant improvement in performance over time. The top curve in Fig. 1 shows Group C's performance over the 80 trials in blocks of 10 trials. A subjects by blocks-of-10-trials analysis of variance showed that Group $\mathrm{C}$ produced a significant decrease in errors over the 4 days of testing $(F=5.76 ; \mathrm{df}=7,21 ; \mathrm{p}<.01)$.

Since the $S s$ in Group CR received 80 trials irrespective of the number of reversals performed, these Ss varied in the total number of complete reversals performed. The fewest number of complete reversals was 6 and the greatest was 10 . The middle curve in Fig. 1 shows the performance of Group CR for the first criterion and the following six reversals. A subjects by reversals analysis of variance for the first criterion and following six reversals also demonstrated a significant progressive improvement over days ( $F=4.44$; df $=6,18 ; p<.01$ ).

The Ss in Group TR were given two reversals per day for 6 days and a subjects by days by reversals-within-days analysis of variance was conducted on these data. Group TR showed no significant improvement in habit reversal over days, but the second reversal per day was performed with significantly fewer errors than the first $(F=4.81 ; \mathrm{df}=1,15 ; \mathrm{p}<.05)$ as seen in Fig. 1.

Running animals in squads helped to avoid one possible artifactual explanation of these data. It might be possible that residual heat from previous trials would serve as a cue to guide present performance. Inspection of the data showed this not to be the case in the present study.

\section{DISCUSSION}

It can be concluded that under the conditions of this study, sow bugs demonstrated successive reversal learning. Compared to the Thompson (1957) study, the above results suggest three possible conclusions. First, it may be argued that the type of reinforcer used is important in assessing learning ability. Since a great deal of an Isopod's behavior is determined by various kinetic and taxic responses(Abbott, 1918; Gunn, 1937; Waterman, 1961), it would seem that a reinforcer (heat) that directly elicited one of these innate responses would produce greater changes in behavior than a stimulus (shock) not normally encountered.

Secondly, the criterion for reversal may be an important factor in obtaining reversal learning. Further clarification of this point is the problem of additional research employing a range of criteria.

Finally, the amount and the schedule of daily training appears to have an effect on reversal learning. Sow bugs may need more than eight trials per day to show successive improvement. Also, the differences between Groups CR and TR, in terms of improvement over days, would suggest that sow bugs need rather intensive practice early in training. The Ss in Group CR did not reach their first criterion until late in Day 1 or early in Day 2 (after 20-30 trials). From then on performance improved fairly rapidly. Group TR was required to start learning to reverse midway through Day 1 (after 10 trials) and to reverse after a given number of trials independently of performance. The better performance on the second reversal per day suggests that the $S s$ in Group TR were learning to respond on a day-to-day basis, while the Ss in Group CR showed significant improvement over days.

\section{REFERENCES}

ABBOTT, C. H. Reaction of land isopods to light. Journal of Experimental Zoology, 1918, 27, 193-246.

FROST, J. The amateur scientist. Scientific American, 1967, May, 142-143.

GUNN, D. L. The humidity reactions of the wood-louse, Porcellio scaber (latr.). Journal of Experimental Biology, 1937, 14, 178-186.

RATNER, S. C., \& DENNY, M. R. Comparative psychology: Research in animal behavior. Homewood, Ill.: Dorsey Press, 1964.

THOMPSON, R. Successive reversal of a position habit in an invertebrate. Science, 1957, 126, 163-164.

WATERMAN, T. H. The physiology of crustacea. Vol. 2. New York: Academic Press, 1961. NOTE

1. Based on a paper read at the Southeastern Psychological Association Convention, New Orleans, La., March 1, 1969. Typing of the manuscript was supported by PHS Research Grant MH12099 from the National Institute of Mental Health. 